Short note

\title{
IgG response against infective larvae of Dirofilaria immitis in experimentally infected cats
}

\author{
Guadalupe PRIETO ${ }^{\mathrm{a}}$, John W. MCCALL ${ }^{\mathrm{b}}$, Luigi VENCO $^{\mathrm{c}}$, \\ Marco GeNCHI ${ }^{\mathrm{d}}$, Fernando SIMÓN ${ }^{\mathrm{a}}$, Claudio GENCHI ${ }^{\mathrm{d} *}$ \\ a Laboratorio de Parasitología, Facultad de Farmacia, Avda. Campo Charro s/n, \\ 37007 Salamanca, Spain \\ ${ }^{\mathrm{b}}$ College of Veterinary Medicine, The University of Georgia, Athens, GA, USA \\ ${ }^{\mathrm{c}}$ Clinica Veterinaria Città di Pavia, Viale Cremona 179, 90133 Pavia, Italy \\ ${ }^{\mathrm{d}}$ Istituto di Patologia Generale Veterinaria, Facoltà di Medicina Veterinaria, Via Celoria 10, \\ 20133 Milano, Italy
}

(Received 17 April 2000; accepted 12 October 2000)

\begin{abstract}
Somatic antigens from third stage larvae of Dirofilaria immitis (SL3) were used to detect IgG response against heartworm infection in 8 experimentally infected cats. A moderate specific anti-SL3 IgG response was found one month post-infection. Afterwards, antibodies decreased reaching a basal level 4 months post-infection and remained at this level until the end of the study, 6 months post-infection. Western blot analysis showed specific recognition of polypeptides of 79,73 , $60,52,40$ and $39 \mathrm{kDa}$ by sera from infected cats 1 month post-infection, but not by sera taken prior to the infection. The low antigenicity of the SL3 antigen in the cat should allow the parasite to escape the host's immune response.
\end{abstract}

cat / heartworm infection / L3 antigens / IgG response

Résumé - Réponse en IgG contre les larves infectieuses de Dirofilaria immitis chez des chats expérimentalement infectés. Des antigènes somatiques provenant de larves du troisième stade de Dirofilaria immitis (SL3) ont été utilisés afin de détecter une réponse en $\operatorname{IgG}$ contre une infection à Dirofilaria immitis chez huit chats expérimentalement infectés. Une réponse modérée des IgG spécifiques anti-L3 a été détectée un mois après infection. Ensuite, les anticorps ont diminué jusqu'à atteindre un niveau de base 4 mois après infection, et sont restés à ce niveau jusqu'à la fin de l'étude, 6 mois après infection. Une analyse en Western blot a montré une reconnaissance spécifique de polypeptides de 79, 73, 60,52, 40 et $39 \mathrm{kDa}$ par les sérums de chats infectés, un mois après infection, mais pas par des sérums prélevés un mois avant infection. La faible antigénicité de l'antigène SL3 chez le chat devrait permettre au parasite d'échapper à la réponse immunitaire de son hôte.

chat / infection à Dirofilaria immitis / antigène L3 / réponse IgG

* Correspondence and reprints

Tel.: (39) 22666 079; fax: (39) 22364 470; e-mail: claudio.genchi@unimi.it 


\section{INTRODUCTION}

Feline heartworm (Dirofilaria immitis) infection has been diagnosed with increasing frequency in the last years in areas where the disease is endemic in dogs. In cats, the infection is characterised by a low worm burden (often 1-2 worms); microfilaremia is transitory and of low intensity and usually naturally infected cats are amicrofilaremic. The evolution of the disease is variable being generally asymptomatic, although sudden death of asymptomatic cats is relatively frequent $[1,5]$. In cats, a strong $\operatorname{IgG}$ response to adult worm antigens is observed and different polypeptides ranging between 19 and $40 \mathrm{kDa}$ are recognized by Western blot [7]. This response is detectable quite early postinfection (2-3 months) [8]. No data are however available on the immune response to third-stage larvae in cats. Because the survival of the infective stage is crucial for the establishment of the infection, preliminary data of the immune response against L3somatic antigens in D. immitis experimentally-infected cats are presented.

\section{MATERIALS AND METHODS}

D. immitis infective larvae (L3) were obtained from experimentally infected Aedes aegypti as described by McCall [4].

Eight cats were infected by subcutaneous injection of the $30 \mathrm{~L} 3 / \mathrm{cat}$ in the lateral region of the neck. Blood samples were obtained prior to infection (time 0 ) from only 3 cats, and 1, 2, 4 and 6 months post-infection from all of the 8 cats. After seven months postinfection, all cats were found to be infected with 1 to 4 worms.

Somatic L3 antigen (SL3) preparation was performed as follows. Infective larvae were sonicated by 4 cycles of $70 \mathrm{kHz}(1 \mathrm{~min}$ each) at $4{ }^{\circ} \mathrm{C}$ and centrifuged at $10000 \mathrm{~g}$ for $20 \mathrm{~min}$. Most of the supernatant was discarded and the resuspended pellet was concentrated by ultrafiltration at $6000 \mathrm{~g}$. The protein concentration was adjusted at $0.5 \mu \mathrm{g} \cdot \mu \mathrm{L}^{-1}$ and stored at $4{ }^{\circ} \mathrm{C}$ until use.
ELISA and Western blot analyses were performed as described by Prieto et al. [7] with some modifications. ELISA polystyrene plates were coated with SL3 antigen at a concentration of $0.5 \mu \mathrm{g} \cdot \mu \mathrm{L}^{-1}$. Sera were employed at a single dilution of 1:200 or at serial dilutions of 1:50, 1:100, 1:200 and $1: 400$, and anti-IgG-peroxidase at 1:4000 dilution. The cut-off point (OD 0.462) was obtained as the OD arithmetic means plus three standard deviations for 43 sera from clinically healthy cats, serologically negative for antibodies against $D$. immitis by a commercially available in-clinic test (Heska ${ }^{\mathrm{TM}}$ Solo Step ${ }^{\mathrm{TM}}$ FH, Heska Corporation, Fort Collins, Colorado, USA), confirmed by an experimental ELISA with somatic and excretory/secretory antigens [7]. In Western blot, pooled sera were employed at a 1:50 dilution and the anti-IgG-peroxidase at a 1:500 dilution.

The statistical analysis was performed by one-way ANOVA and a 5\% level of significance $(p \leq 0.05)$ was used to assess statistical differences.

\section{RESULTS}

Specific anti-SL3 IgG was detected at moderate levels $(\mathrm{OD}=0.6)$ only 1 month

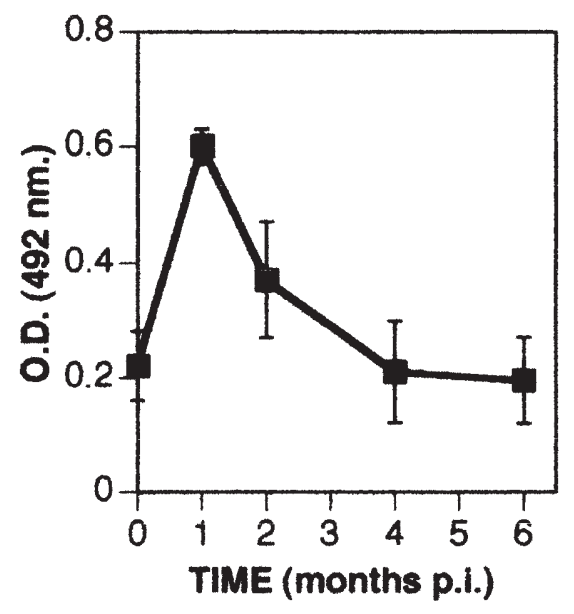

Figure 1. Antibody response (mean ODs) to SL3 antigens in 8 experimentally infected cats. 

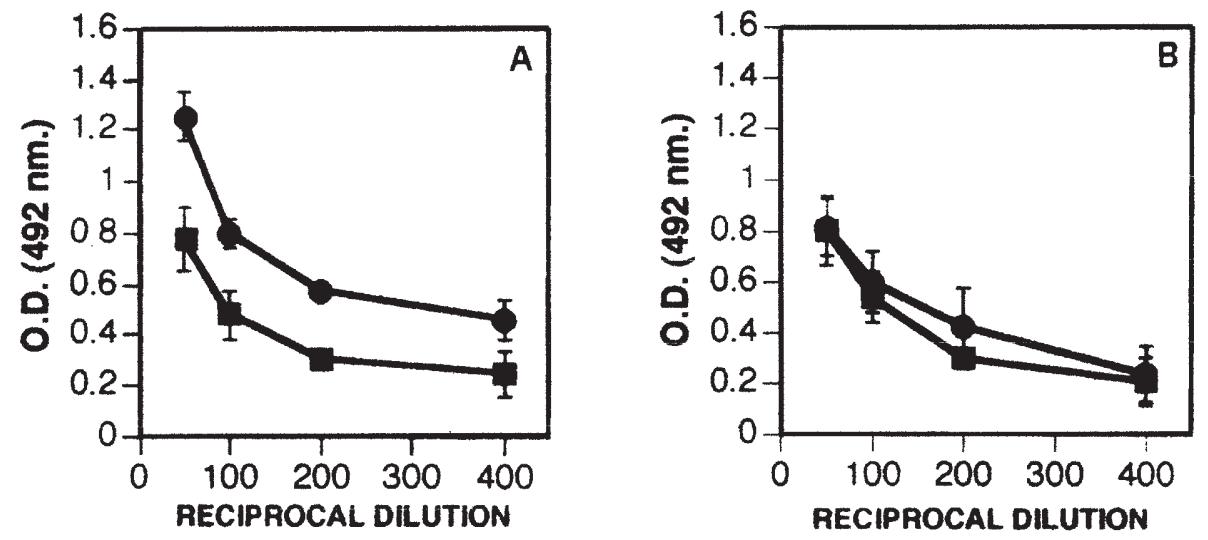

Figure 2. Mean OD obtained by ELISA analysis with the SL3 antigen from sera from 3 experimentally infected cats obtained prior to infection and from 8 cats 1 and 2 months post-infection. A. ELISA with sera from cats prior to infection $(\mathbf{\square})$ and 1 month post-infection $(\mathbf{O})$. B. ELISA with sera from cats prior to infection ( $\mathbf{\square}$ ) and 2 months post-infection $(\boldsymbol{O})$. Standard deviation bars are shown.

post-infection; afterwards the antibodies decreased, reaching a basal level 4 months post-infection and remaining at this level until the end of the study (Fig. 1). A comparison of the ODs of sera from 1 month post-infection and time 0 indicated that all of the tested dilutions were significantly different. No differences were found between time 0 and 2 months post-infection (Fig. 2). Western blot analysis showed specific recognition of polypeptides of $79,73,60,52,40$ and $39 \mathrm{kDa}$ by sera from infected cats 1 month post-infection, but not by sera taken prior to infection (time 0 ) and by serum from a cat infected with Ancylostoma tubaeforme (Fig. 3).

\section{DISCUSSION}

Our results, though obtained from a low number of experimentally infected cats, showed that the immune response against L3-somatic antigens in cats is weak, of short duration and able to recognize a low number of antigens. Previously, a strong IgG response against adult worm antigens was

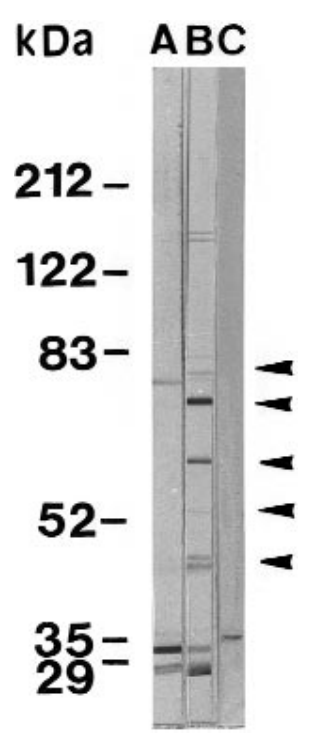

Figure 3. Western blot analysis of the SL3 antigen with a pool of sera from 3 cats prior to infection (A), a pool of sera from 3 cats 1 month postinfection $(\mathbf{B})$ and serum from a cat infected with Ancylostoma tubaeforme. 
found from the 2 nd-3rd month post-infection on, probably as a consequence of common antigens shared both by 4th stage larvae, preadult and adult worms $[7,8]$.

In areas endemic for canine heartworm infection, the seroprevalence of IgG antibodies against the parasite is quite high in cats $(44 \%)$ with respiratory and gastrointestinal signs [9]. Furthermore, in an extensive survey involving more that 2000 cats, who were mostly asymptomatic, Miller et al. [6] found that the overall risk of exposure to heartworm infection was about $12 \%$, ranging from 5-33\%. It is thought however that many cats spontaneously recover and no clinical signs of infection are observed. Because the immune response against the infective stage seems to be quite low and of short duration, the parasite can probably develop to the 4th larval stage. The strong immune response detectable at the 4th month post-infection [7, 8], is likely to interfere with the development of the parasite at the adult stage. The low level of antiSL3 IgG antibody and the short period in which they are detectable should be due to the short life span of this stage (3-9 days) [3]. Furthermore, our results seem to confirm the observations of Ibrahim et al. [2]. In this study, both in vitro and in vivo $D$. immitis L3 were found to shed many surface antigens (mostly of 6 and $35 \mathrm{kDa}$ ) which were not replaced. This loss of surface peptides resulted in a reduction in the antigenic potential of infective larvae. We therefore conclude that shedding of surface peptides and reducing surface antigenicity may represent mechanisms by which $D$. immitis infective larvae evade the host immune attack. Because the immune response to L3 larvae seems to be critical, it is possible to speculate that antigens from the L4 larvae may be better able to induce protective immunity than extracts from the L3 stage. The study of immune response to L4 extracts should validate this hypothesis.

\section{ACKNOWLEDGEMENTS}

This study was supported by a EC contract BMH4-CT98-9578 (DG-SSMI) and MURST 1999, prot. 9907151238.

\section{REFERENCES}

[1] Genchi C., Kramer L., Venco L., Prieto G., Simon F., Comparison of antibody and antigen testing with echocardiography for the detection of heartworm (Dirofilaria immitis) in cats, in: Seward R.L., Knight D.H. (Eds.), Recent advances in Heartworm Disease: Symposium '98, Batavia, IL, American Heartworm Society, 1998, pp. 173177

[2] Ibrahim M.S., Tamashiro W.R., Moraga D.A., Scott A.L., Antigen shedding from the surface of the infective stage larval of Dirofilaria immitis, Parasitology 99 (1989) 89-97.

[3] Kotani T., Powers K.G., Developmental states of Dirofilaria immitis in the dog, Am. J. Vet. Res. 43 (1982) 2199-2206.

[4] McCall J.W., The role of arthropods in the development of animal models for filariasis research, J. Georgia Entomol. Soc. 16 (1981) 283-293.

[5] McCall J.W., Calvert C.A., Rawlings C.A., Heartworm infection in cats: A life-threating disease, Vet. Med. 89 (1994) 639-647.

[6] Miller M.W., Atkins C.E., Stemme K., RobertsonPlouch C., Guerrero J., Prevalence of exposure to Dirofilaria immitis in cats in multiple areas of the United States, in: Seward R.L., Knight D.H. (Eds.), Recent advances in Heartworm Disease: Symposium '98, Batavia, IL, American Heartworm Society, 1998, pp. 161-166.

[7] Prieto C., Genchi C., Simón F., Venco L., Feline heartworm (Dirofilaria immitis) infection: detection of specific IgG for the diagnosis of occult infections, Vet. Parasitol. 70 (1997) 209-217.

[8] Prieto G., Genchi C., McCall J.W., Simón F., Venco L., Utility of adult antigens of Dirofilaria immitis for the early detection of dirofilariosis and for the evaluation of chemoprophylactic treatment in experimentally infected cats, Vet. Parasitol. 86 (1999) 5-13.

[9] Robertson-Plouch C.K., Dillon A.R., Brawner W.R., Guerrero J., Prevalence of feline heartworm infection among cats with respiratory and gastrointestinal signs: results of a multicenter study, in: Seward R.L., Knight D.H. (Eds.), Recent advances in Heartworm Disease: Symposium '98, Batavia, IL, American Heartworm Society, 1998, pp. 161-166. 